\title{
Reaction of 1-Alkylthioisoquinolinium Salts with Active Methylene Compounds
}

\author{
Reiko FuJITA, * Noriyuki Watanabe, and Hiroshi TomisaWA \\ Tohoku Pharmaceutical University, 4-4-1 Komatsushima, Aoba-ku, Sendai 981-8558, Japan. \\ Received September 10, 2001; accepted October 23, 2001
}

\begin{abstract}
2-Alkyl-1-alkylthioisoquinolinium salts were readily prepared from 2-alkyl-1(2H)-isoquinolones via 2-alkyl$1(2 \mathrm{H})$-thioisoquinolones in two steps. Under mild conditions, the reaction of 2 -alkyl-l-alkylthioisoquinolinium salts with active methylene compounds in the presence of sodium hydride afforded 2-alkyl-1-(substituted methylene)iso-quinolines in good yields. Pyrrolo[2,1-a]isoquinolines were synthesized by the cyclization of 2-benzyl-1(substituted methylene)isoquinolines using acetic anhydride.
\end{abstract} pound

Key words cyclization; pyrrolo[2,1-a]isoquinoline; dimethyl malonate; methylthioisoquinolinium salt; active methylene com-

We have previously reported on the reaction of 2methylthioquinolinium salts with active methylene compounds for the formation of a carbon-carbon bond. ${ }^{1)}$ Additionally, various methods for the synthesis of indolizine derivatives have been reported. ${ }^{2)}$ In view of the recent report by Iwao and Kuraishi. ${ }^{2)}$ on the novel total synthesis of the marine alkaloid lamellarins, which contain the pyrrolo[2,1a]isoquinoline skeleton (Chart 1), ${ }^{3)}$ functionalized pyrrolo$[2,1-a]$ isoquinolines would function as very useful synthetic intermediates toward their syntheses. The conventional methods for preparing the substituted pyrrolo[2,1- $a$ ] isoquinoline derivatives are either via Michael condensation of Reissert compounds, ${ }^{4)}$ or via 1,3- and 1,5-dipolar cycloaddition reactions. ${ }^{5,6)}$ In this paper, we report a novel method for the synthesis of a pyrrolo[2,1- $a$ ] isoquinoline skeleton using the cyclization of 2-benzyl-1-(substituted methylene) isoquinolines, which are readily prepared by the reaction of 2-benzyl1-alkylthioisoquinolinium iodides with active methylene compounds.

Reaction of 1-Alkylthioisoquinolinium Iodides with Active Methylene Compounds Initially, the reaction of active methylene compounds with isoquinolinium salts that have a methylthio group as a leaving group at the 1-position was examined under mild conditions in the presence of sodium hydride (Chart 2, Table 1). 2-Methyl- and 2-benzyl-1(2H)thioisoquinolones $\left(3,{ }^{7}\right)$ 4) were prepared from 2-methyl- and 2-benzyl-1 $(2 H)$-isoquinolones $\left(\mathbf{1},{ }^{8)} \mathbf{2}\right)$ in excellent yields, respectively. The reaction of thioisoquinolones $(3,4)$ with methyl iodide (5a) afforded 2-methyl- and 2-benzyl-1methylthioisoquinolinium iodides $(6 \mathbf{6}, 9)$ 7) in 99 and $77 \%$ yields, respectively. The reactions of $\mathbf{6 a}$ with straight-chain active methylene compounds $(\mathbf{8 a}-\mathbf{c})$ in the presence of sodium hydride for $1.5 \mathrm{~h}$ at room temperature afforded 2methyl-1-(substituted methylene)isoquinolines (9a, 88\%; 9b, $81 \%$; 9c, $85 \%$, respectively) in high yields. However, reactions of $\mathbf{6 a}$ with straight-chain active methylene compounds $(\mathbf{8 d}-\mathbf{f})$ afforded $9 \mathbf{d}-\mathbf{f}$ in moderate yields. Reactions of $\mathbf{6 a}$ with cyclic active methylene compounds $(\mathbf{8 g}, \mathbf{h})$ at $90^{\circ} \mathrm{C}$ for $3 \mathrm{~h}$ gave 2-methyl-1-(substituted methylene)isoquinolines $(\mathbf{9 g}, \mathbf{h})$ in $92 \%$ and $61 \%$ yields, respectively. Similarly, the reaction of 7 with $\mathbf{8 a}$ - e produced 2-benzyl-1-(substituted methylene)-isoquinolines (10a, 95\%; 10b, 98\%; 10c, 99\%; 10d, $88 \%$; 10e, $53 \%$, respectively). To examine the effects of the alkylthio group at the 1-position, the experiments were repeated using other alkyl iodides. In comparison to methyl iodide, the reaction of $\mathbf{3}$ with ethyl iodide (5b) and isopropyl iodide (5c) afforded low yields of the corresponding 1alkylthioisoquinolinium salts $(6 \mathbf{b}, 75 \%$; 6c, 52\%). Furthermore, the reactions of $\mathbf{6 b}, \mathbf{c}$ with $\mathbf{8 a}$ and with $\mathbf{8 g}$ gave products [9a $(65 \%, 65 \%) ; 9 g(42 \%, 40 \%)]$ in lower yields than that of $6 \mathbf{a}$ with $8 \mathbf{a}, \mathbf{g}$ (Table 1). These results can be explained by assuming that the enol form arising from the active methylenes $(\mathbf{8 d}-\mathbf{f})$ would be more stable than the corresponding keto form, and would tend to lower the yields. The reactions of 7 with bulky methylene compounds $(\mathbf{8 f}-\mathbf{h})$ did not give the desired products $(\mathbf{1 0 f}-\mathbf{h})$, whereas reactions of $\mathbf{6 a}$ bearing a smaller methyl group at the 2-position proceeded with $\mathbf{8 f - h}$; hence it can be implied that steric hindrance between two bulky groups, specifically, 2-benzyl and phenyl in $\mathbf{8 f}$ or alicyclic rings $(\mathbf{8 g}, \mathbf{h})$ was a main factor in reducing the yield. Spectroscopic studies of compound 10b showed nuclear Overhauser and exchange spectroscopy (NOE) correlations between the methyl of the ester and the 2-methylene groups, and therefore their configuration is suggested to be the Zform. Unfortunately, similar NOE correlations were not observed between the ester and the 2-substituent groups of $\mathbf{9 b}, \mathbf{d}$, and $\mathbf{1 0 d}$. In summary, this type of reaction is a promising method to form a carbon-carbon bond at the 1-position of an isoquinoline ring.

Synthesis of Pyrrolo[2,1-a]isoquinolines Our next step was cyclization of the 2-benzyl compounds $(\mathbf{1 0 a}-\mathbf{e})$ in acetic anhydride to provide the functionalized pyrrolo[2,1a] isoquinoline derivatives (Chart 3). Heating of 10a, $\mathbf{d}, \mathbf{e}$ in acetic anhydride at $130{ }^{\circ} \mathrm{C}$ for $4 \mathrm{~h}$ afforded the corresponding pyrrolo[2,1-a] isoquinolines (11a, 91\%; 11b, 88\%; 11c, 68\%; respectively). Unfortunately, cyclizations of $\mathbf{1 0 b}, \mathbf{c}$ were unsuccessful, although $\mathbf{1 0 b}, \mathbf{c}$ were recovered. The structures of 11a - c were determined as follows. ${ }^{1} \mathrm{H}-\mathrm{NMR}$ spectra of $11 \mathrm{a}-\mathrm{c}$ showed that the signals $(5.55-5.94 \mathrm{ppm})$ due to
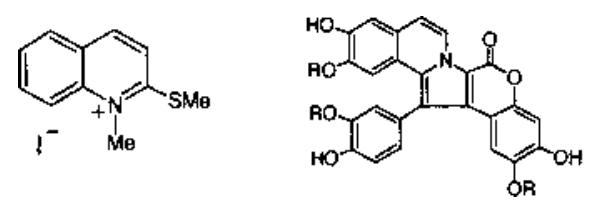

$\mathrm{P}=$ Ma lamollatin $\mathrm{D}$ $\mathrm{A}=\mathrm{H}$ lamelarin $\mathrm{H}$

Chart 1 

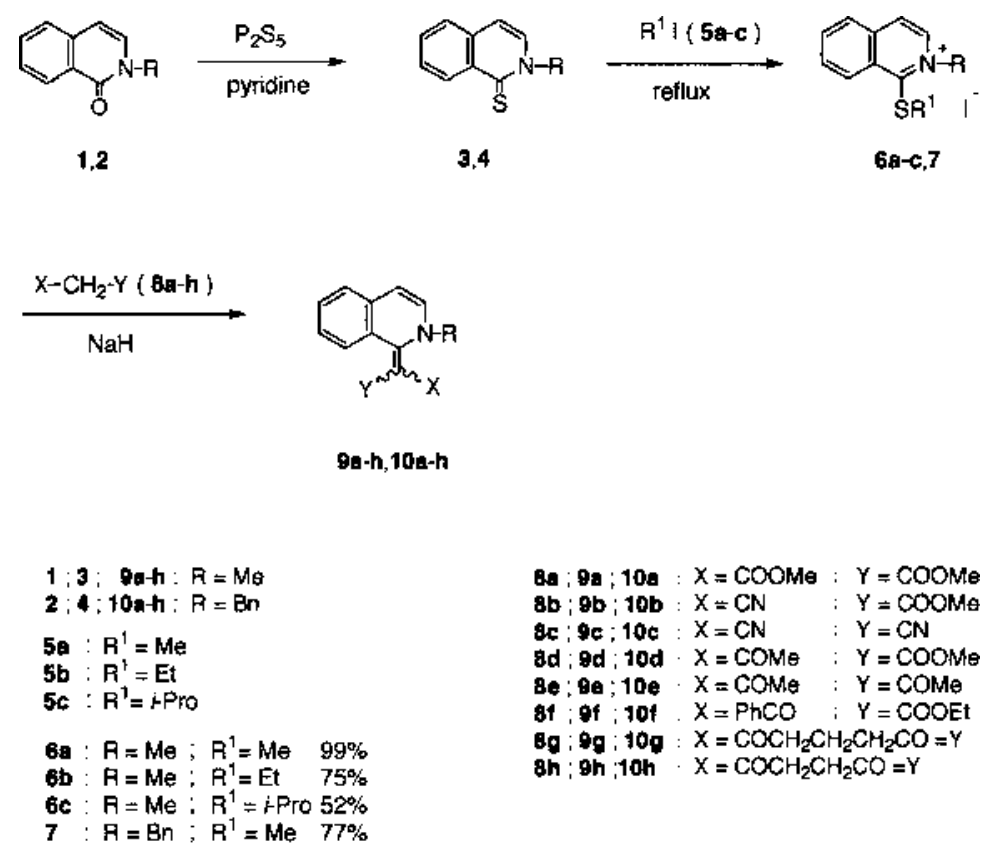

Chart 2

Table 1. Reaction of Isoquinolinium Salts with Active Methylene Compounds

\begin{tabular}{|c|c|c|c|c|c|c|c|c|}
\hline \multirow{2}{*}{ Entry } & \multirow{2}{*}{ Salt } & \multirow{2}{*}{$\begin{array}{l}\text { Temp } \\
\left({ }^{\circ} \mathrm{C}\right)\end{array}$} & \multirow{2}{*}{$\begin{array}{l}\text { Time } \\
\text { (h) }\end{array}$} & \multirow{2}{*}{ Solvent } & \multicolumn{2}{|c|}{$8 a-h$} & \multirow{2}{*}{ Product } & \multirow{2}{*}{$\begin{array}{c}\text { Yield } \\
(\%)\end{array}$} \\
\hline & & & & & $\mathrm{X}$ & $\mathrm{Y}$ & & \\
\hline 1 & $6 a$ & $\mathrm{rt}^{a)}$ & 1.5 & THF & COOMe & COOMe & $9 a$ & 97 \\
\hline 2 & $\mathbf{6 a}$ & $\mathrm{rt}$ & 1.5 & THF & $\mathrm{CN}$ & COOMe & $9 \mathbf{b}$ & 81 \\
\hline 3 & $6 a$ & $\mathrm{rt}$ & 1.5 & THF & $\mathrm{CN}$ & $\mathrm{CN}$ & $9 c$ & 85 \\
\hline 4 & $6 a$ & $\mathrm{rt}$ & 1.5 & THF & $\mathrm{COMe}$ & COOMe & 9d & 69 \\
\hline 5 & $6 \mathbf{a}$ & $\mathrm{rt}$ & 1.5 & THF & $\mathrm{COMe}$ & $\mathrm{COMe}$ & $9 e$ & 47 \\
\hline 6 & $6 a$ & $\mathrm{rt}$ & 1.5 & THF & $\mathrm{PhCO}$ & COOEt & $9 f$ & 49 \\
\hline 7 & $6 a$ & 90 & 3 & DMF & \multicolumn{2}{|c|}{$\mathrm{COCH}_{2} \mathrm{CH}_{2} \mathrm{CH}_{2} \mathrm{CO}$} & $9 \mathrm{~g}$ & 92 \\
\hline 8 & $6 \mathbf{a}$ & 90 & 3 & DMF & \multicolumn{2}{|c|}{$\mathrm{COCH}_{2} \mathrm{CH}_{2} \mathrm{CO}$} & $9 \mathrm{~h}$ & 61 \\
\hline 9 & 7 & $\mathrm{rt}$ & 2 & THF & COOMe & COOMe & $10 \mathrm{a}$ & 95 \\
\hline 10 & 7 & $\mathrm{rt}$ & 2 & THF & $\mathrm{CN}$ & $\mathrm{COOMe}$ & $10 \mathrm{~b}$ & 98 \\
\hline 11 & 7 & $\mathrm{rt}$ & 2 & THF & $\mathrm{CN}$ & $\mathrm{CN}$ & $10 \mathrm{c}$ & 99 \\
\hline 12 & 7 & $\mathrm{rt}$ & 2 & THF & $\mathrm{COMe}$ & COOMe & 10d & 88 \\
\hline 13 & 7 & $\mathrm{rt}$ & 2 & THF & $\mathrm{COMe}$ & $\mathrm{COMe}$ & $10 \mathrm{e}$ & 53 \\
\hline 14 & 7 & $\mathrm{rt}$ & 2 & THF & $\mathrm{PhCO}$ & COOEt & $10 \mathrm{f}$ & 0 \\
\hline 15 & 7 & 90 & 3 & DMF & \multicolumn{2}{|c|}{$\begin{array}{c}\mathrm{COCH}_{2} \mathrm{CH}_{2} \mathrm{CH}_{2} \mathrm{CO} \\
\mathrm{COCH}_{2} \mathrm{CH}_{2} \mathrm{CO}\end{array}$} & $10 \mathrm{~g}$ & 0 \\
\hline 16 & 7 & 90 & 3 & DMF & \multicolumn{2}{|c|}{$\mathrm{COCH}_{2} \mathrm{CH}_{2} \mathrm{CO}$} & $10 \mathrm{~h}$ & 0 \\
\hline 17 & $\mathbf{6 b}$ & $\mathrm{rt}$ & 1.5 & THF & COOMe & $\mathrm{COOMe}$ & $9 a$ & 65 \\
\hline 18 & $6 c$ & $\mathrm{rt}$ & 1.5 & THF & COOMe & COOMe & $9 a$ & 59 \\
\hline 19 & $6 b$ & 90 & 3 & DMF & \multicolumn{2}{|c|}{$\mathrm{COCH}_{2} \mathrm{CH}_{2} \mathrm{CH}_{2} \mathrm{CO}$} & $9 g$ & 42 \\
\hline 20 & $6 c$ & 90 & 3 & DMF & \multicolumn{2}{|c|}{$\mathrm{COCH}_{2} \mathrm{CH}_{2} \mathrm{CH}_{2} \mathrm{CO}$} & $9 \mathrm{~g}$ & 40 \\
\hline
\end{tabular}

a) Room temperature.

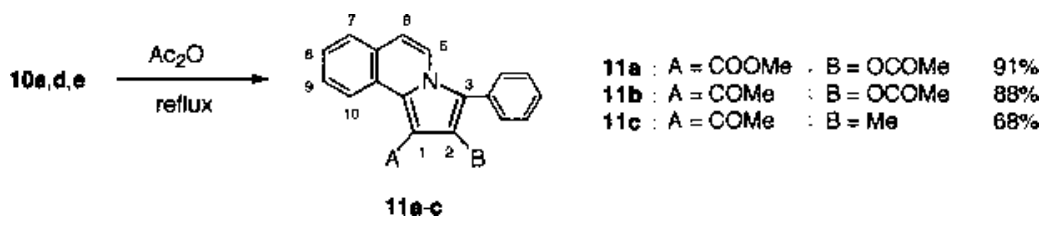

Chart 3

methylenes of benzyl groups in 10a, $\mathbf{d}, \mathbf{e}$ disappeared by dehydration and condensation.

In conclusion, we found that a novel reaction of active methylene compounds with isoquinolinium salts having a methylthio group as a leaving group at the 1-position gave 2- alkyl-1-(substituted methylene)isoquinolines under mild condition in fairly good yields. Cyclization of 2-benzyl compounds having an ester or an acetyl group in acetic anhydride produced the pyrrolo[2,1-a] isoquinolines in excellent yields. 


\section{Experimental}

The following instruments were used to obtain physical data: Melting points, Yanaco micromelting point apparatus (values are uncorrected); IR spectra, Perkin-Elmer ET-IR1725X spectrometer; MS, JEOL JMN-DX 303/JMA-DA 5000 spectrometer; NMR spectra, JNM-GSX 400 ( ${ }^{1} \mathrm{H}-\mathrm{NMR}$, $\left.400 \mathrm{MHz} ;{ }^{13} \mathrm{C}-\mathrm{NMR}, 100 \mathrm{MHz}\right), \mathrm{JNM}-\mathrm{EX} 270\left({ }^{1} \mathrm{H}-\mathrm{NMR}, 270 \mathrm{MHz} ;{ }^{13} \mathrm{C}-\right.$ NMR, $67.8 \mathrm{MHz}$ ), JEOL JNM- PMX $60_{\mathrm{SI}}$ spectrometer with tetramethylsilane (TMS) as an internal standard; elemental analyses, Perkin-Elmer 2400 CHN Elemental Analyzer. The following experimental conditions were used for chromatography; column chromatography, Merck Kieselgel silica gel 60 (230 - $400 \mathrm{mesh})$; TLC, pre-coated TLC plates with $60 \mathrm{~F}_{254}(2 \mathrm{~mm}$, Merck).

Synthesis of 2 An ethanol solution $(20 \mathrm{ml})$ of $\mathrm{KOH}(0.59 \mathrm{~g}, 10.5 \mathrm{mmol})$, isoquinolone $(1.45 \mathrm{~g}, 10 \mathrm{mmol})$, and benzyl chloride $(1.35 \mathrm{~g}, 10.5 \mathrm{mmol})$ was heated at $100{ }^{\circ} \mathrm{C}$ for $10 \mathrm{~h}$ in a sealed tube. After removing the solvent in vacuo, the residue was washed with hexane and was recrystallized from isopropyl ether to afford 2 as colorless needles $(2.33 \mathrm{~g}, 99 \%)$ : $\mathrm{mp} 70^{\circ} \mathrm{C}$ (isopropyl ether). IR $\left(\mathrm{CHCl}_{3}\right) \mathrm{cm}^{-1}: 1650,1599,741,692 .{ }^{1} \mathrm{H}-\mathrm{NMR}\left(\mathrm{CDCl}_{3}\right) \delta$ : $5.22\left(2 \mathrm{H}, \mathrm{s}, \mathrm{CH}_{2}\right), 6.47(1 \mathrm{H}, \mathrm{d}, J=7.4 \mathrm{~Hz}, \mathrm{H}-4), 7.08(1 \mathrm{H}, \mathrm{d}, J=7.4 \mathrm{~Hz}, \mathrm{H}-3)$, $7.24-7.39(5 \mathrm{H}, \mathrm{m}, \mathrm{H}-\mathrm{Ph}), 7.46-7.65(3 \mathrm{H}, \mathrm{br} \mathrm{m}, \mathrm{H}-5,6,7), 8.46(1 \mathrm{H}, \mathrm{dd}$, $J=1.2,7.2 \mathrm{~Hz}, \mathrm{H}-8) .{ }^{13} \mathrm{C}-\mathrm{NMR}\left(\mathrm{CDCl}_{3}\right) \delta: 51.65,106.38,125.89,126.32$, 126.87, 127.79 (C2), 127.94, 128.06, 128.78 (C2), 131.27, 132.19, 136.90, 136.97, 162.24. MS $m / z: 235\left(\mathrm{M}^{+}\right)$. High resolution (HR)-MS $m / z$ : Calcd for $\mathrm{C}_{16} \mathrm{H}_{13} \mathrm{NO}, 235.0997$. Found: 235.0985.

Synthesis of 4 A solution of $2(0.47 \mathrm{~g}, 2 \mathrm{mmol})$ and phosphorus pentasulfide $(0.467 \mathrm{~g}, 2.1 \mathrm{mmol})$ in pyridine $(6 \mathrm{ml})$ was heated at $150^{\circ} \mathrm{C}$ for $5 \mathrm{~h}$. The reaction mixture was mixed with water $(10 \mathrm{ml})$ and the resulting solution was extracted with $\mathrm{CHCl}_{3}$. The $\mathrm{CHCl}_{3}$ layer was dried over $\mathrm{MgSO}_{4}$ and evaporated. The residue was recrystallized from isopropyl ether to afford 4 as colorless needles $(0.49 \mathrm{~g}, 97 \%)$ : mp $95-97^{\circ} \mathrm{C}$. IR $\left(\mathrm{CHCl}_{3}\right) \mathrm{cm}^{-1}: 1547$, 1290, 773, 694. ${ }^{1} \mathrm{H}-\mathrm{NMR}\left(\mathrm{CDCl}_{3}\right) \delta: 6.01\left(2 \mathrm{H}, \mathrm{s}, \mathrm{CH}_{2}\right), 6.87(1 \mathrm{H}, \mathrm{d}$, $J=7.1 \mathrm{~Hz}, \mathrm{H}-4), 7.31-7.34(5 \mathrm{H}, \mathrm{m}, \mathrm{H}-\mathrm{Ph}), 7.40(1 \mathrm{H}, \mathrm{d}, J=7.1 \mathrm{~Hz}, \mathrm{H}-3)$, $7.54-7.61(2 \mathrm{H}$, br m, H-5, 7), 7.67 (1H, ddd, $J=1.5,7.5,7.5 \mathrm{~Hz}, 6-\mathrm{H}), 9.16$ $(1 \mathrm{H}, \mathrm{dd}, J=1.5,7.5 \mathrm{~Hz}, \mathrm{H}-8) .{ }^{13} \mathrm{C}-\mathrm{NMR}\left(\mathrm{CDCl}_{3}\right) \delta: 59.26,112.42,126.67$, 127.94 (C3), 127.97, 128.55, 128.85 (C2), 132.11, 132.41, 133.05 (C2), 135.76, 184.63. MS $m / z$ : $251\left(\mathrm{M}^{+}\right)$. HR-MS $m / z$ : Calcd for $\mathrm{C}_{16} \mathrm{H}_{13} \mathrm{NS}$, 251.0769. Found: 251.0727 .

General Procedure for Synthesis of 2-Alkyl-1-alkylthioisoquinolinium Iodides (6a-c, 7) A solution of $4(0.5 \mathrm{~g}, 2 \mathrm{mmol}), \mathbf{5 a}(1.42 \mathrm{~g}, 10 \mathrm{mmol})$, and benzene $(7 \mathrm{ml})$ was gently refluxed for $17 \mathrm{~h}$. The resulting yellow precipitate was collected by filtration and was recrystallized from methanol to give $7(0.6 \mathrm{~g}, 77 \%)$. Reactions of $\mathbf{3}$ with $\mathbf{5 a}-\mathbf{c}$ were carried out under similar conditions to afford 6a, 1-ethylthio-(6b), and 1-isopropylthio-2-methylisoquinolinium iodide (6c). Yields are listed in Chart 2.

$\mathbf{6 a}^{9}{ }^{9}$ : Yellow needles (methanol), mp 132-134 ${ }^{\circ} \mathrm{C}\left(\mathrm{mp} 143-146^{\circ} \mathrm{C}\right) .{ }^{9)}$ ${ }^{1} \mathrm{H}-\mathrm{NMR}\left(\mathrm{DMSO}-d_{6}\right) \delta: 2.83(3 \mathrm{H}, \mathrm{s}, \mathrm{SMe}), 4.66(3 \mathrm{H}, \mathrm{s}, \mathrm{NMe}), 8.0-8.39$ $(3 \mathrm{H}, \mathrm{m}, \mathrm{H}-5,6,7), 8.75(1 \mathrm{H}, \mathrm{d}, J=7.0 \mathrm{~Hz}, \mathrm{H}-4), 8.85(1 \mathrm{H}, \mathrm{dd}, J=2.0$, $6.0 \mathrm{~Hz}, \mathrm{H}-8), 9.0(1 \mathrm{H}, \mathrm{d}, J=7.0 \mathrm{~Hz}, \mathrm{H}-3) .{ }^{13} \mathrm{C}-\mathrm{NMR}\left(\mathrm{CDCl}_{3}\right) \delta: 20.10$, $48.77,125.08,128.17,129.71,130.10,131.85,136.00,136.90,138.22$, 160.87 .

6b: Yellow needles (methanol), $\mathrm{mp} 104^{\circ} \mathrm{C}$. IR $\left(\mathrm{CHCl}_{3}\right) \mathrm{cm}^{-1}: 1604,773$, 755. ${ }^{1} \mathrm{H}-\mathrm{NMR}$ (DMSO- $\left.d_{6}\right) \delta: 1.36(3 \mathrm{H}, \mathrm{dd}, J=7.0,7.0 \mathrm{~Hz}, \mathrm{CMe}), 3.40(2 \mathrm{H}$, ddd, $\left.J=7.0,7.0,7.0 \mathrm{~Hz}, \mathrm{CH}_{2}\right), 4.96(3 \mathrm{H}, \mathrm{s}, \mathrm{NMe}), 8.00-8.50(3 \mathrm{H}, \mathrm{m}, \mathrm{H}-5$, 6, 7), $8.54(1 \mathrm{H}, \mathrm{d}, J=7.0 \mathrm{~Hz}, \mathrm{H}-4), 8.87(1 \mathrm{H}, \mathrm{brm}, \mathrm{H}-8), 9.21(1 \mathrm{H}, \mathrm{d}$, $J=7.0 \mathrm{~Hz}, \mathrm{H}-3) .{ }^{13} \mathrm{C}-\mathrm{NMR}\left(\mathrm{CDCl}_{3}\right) \delta: 15.08,32.64,48.90,125.30,128.13$, 129.92, 130.85, 131.91, 136.09, 136.94, 138.48, 159.29. Anal. Calcd for $\mathrm{C}_{12} \mathrm{H}_{14}$ INS: C, 43.52; H, 4.26; N, 4.23. Found: C, 43.33; H, 4.20; N, 4.03 .

6c: Yellow needles (methanol), $\mathrm{mp} 139-140^{\circ} \mathrm{C}$. IR $\left(\mathrm{CHCl}_{3}\right) \mathrm{cm}^{-1}: 1620$, 785, 757. ${ }^{1} \mathrm{H}-\mathrm{NMR}$ (DMSO- $\left.d_{6}\right) \delta: 1.35(6 \mathrm{H}, \mathrm{d}, J=7.0 \mathrm{~Hz}, \mathrm{CMex} 2), 3.91$ $(1 \mathrm{H}, \mathrm{m}, J=7.0 \mathrm{~Hz}, \mathrm{CH}), 4.76(3 \mathrm{H}, \mathrm{s}, \mathrm{NMe}), 8.00-8.63(3 \mathrm{H}, \mathrm{m}, \mathrm{H}-5,6,7)$, $8.73(1 \mathrm{H}, \mathrm{d}, J=7.0 \mathrm{~Hz}, \mathrm{H}-4), 9.00(1 \mathrm{H}, \mathrm{br}, \mathrm{H}-8), 9.09(1 \mathrm{H}, \mathrm{d}, J=7.0 \mathrm{~Hz}$, $\mathrm{H}-3) .{ }^{13} \mathrm{C}-\mathrm{NMR}\left(\mathrm{CDCl}_{3}\right) \delta: 23.43(\mathrm{C} 2), 44.83,48.96,125.57,128.12$, 130.08, 131.33, 132.02, 136.20, 137.06, 138.72, 158.20. Anal. Calcd for $\mathrm{C}_{13} \mathrm{H}_{16} \mathrm{INS}: \mathrm{C}, 45.23 ; \mathrm{H}, 4.67 ; \mathrm{N}, 4.06$. Found: $\mathrm{C}, 45.05 ; \mathrm{H}, 4.37 ; \mathrm{N}, 3.99$.

7: Yellow needles (methanol), $\mathrm{mp} 110^{\circ} \mathrm{C}$. IR $\left(\mathrm{CHCl}_{3}\right) \mathrm{cm}^{-1}: 1618,725$, 696. ${ }^{1} \mathrm{H}-\mathrm{NMR}\left(\mathrm{CF}_{3} \mathrm{COOD}\right) \delta: 2.67(3 \mathrm{H}, \mathrm{s}, \mathrm{SMe}), 6.40\left(2 \mathrm{H}, \mathrm{s}, \mathrm{CH}_{2}\right), 7.32-$ $7.45(5 \mathrm{H}, \mathrm{m}, \mathrm{H}-\mathrm{Ph}), 8.17(1 \mathrm{H}$, ddd, $J=1.3,7.1,8.4 \mathrm{~Hz}, \mathrm{H}-6), 8.30(1 \mathrm{H}$, ddd, $J=1.3,7.1,8.4 \mathrm{~Hz}, \mathrm{H}-7), 8.45(1 \mathrm{H}, \mathrm{dd}, J=1.3,8.4 \mathrm{~Hz}, \mathrm{H}-5), 8.74(1 \mathrm{H}, \mathrm{d}$, $J=6.8 \mathrm{~Hz}, \mathrm{H}-4), 8.87(1 \mathrm{H}, \mathrm{dd}, J=1.3,8.4 \mathrm{~Hz}, \mathrm{H}-8), 9.13(1 \mathrm{H}, \mathrm{d}, J=6.8 \mathrm{~Hz}$, $\mathrm{H}-3) .{ }^{13} \mathrm{C}-\mathrm{NMR}\left(\mathrm{CDCl}_{3}\right) \delta: 21.62,63.24,111.90,126.05,127.51$ (C2), $128.38,128.46,128.96$ (C2), 132.19, 134.62, 136.50, 137.34, 137.33, 137.72, 161.43. Anal. Calcd for $\mathrm{C}_{17} \mathrm{H}_{16} \mathrm{INS}$ C, 51.92; H, 4.10; N, 3.56 . Found: C, 51.76; H, 3.83; N, 3.59.

General Procedure for Reaction of Isoquinolinium Salts $(6 a-c, 7)$ with Active Methylene Compounds $(8 \mathbf{a}-\mathbf{h})$ To a suspension of $\mathrm{NaH}$
( $15 \mathrm{mg}, 0.6 \mathrm{mmol})$ in tetrahydrofuran (THF) $(5 \mathrm{ml})$ was added dimethyl malonate $(79 \mathrm{mg}, 0.6 \mathrm{mmol})$ at $0{ }^{\circ} \mathrm{C}$ under $\mathrm{N}_{2}$. The mixture was stirred for $10 \mathrm{~min}$ at room temperature followed by the addition of $6 \mathrm{a}(165 \mathrm{mg}, 0.5$ $\mathrm{mmol}$ ). The reaction mixture was stirred for $1.5 \mathrm{~h}$ at room temperature, then quenched with water $(5 \mathrm{ml})$ followed by saturated $\mathrm{Na}_{2} \mathrm{~S}_{2} \mathrm{O}_{3}$ solution $(3 \mathrm{ml})$. The reaction mixture was extracted with $\mathrm{CHCl}_{3}$ and dried over $\mathrm{MgSO}_{4}$. The organic layer was concentrated in vacuo to give 1,2-dihydro-1-[bis(methoxycarbonyl)methylene]-2-methylisoquinoline [9a (166 mg, 97\%)]. Reactions of 1-ethylthio-, 1-isopropylthio-2-methylisoquinoline $(\mathbf{6 b}, \mathbf{c})$ and 7 with dimethyl malonate (8a), methyl cyanoacetate $(\mathbf{8 b})$, malononitrile $(\mathbf{8 c})$, methyl acetoacetate $(\mathbf{8 d})$, acetylacetone $(\mathbf{8 e})$, ethyl benzoacetate $(\mathbf{8 f})$, 1,3-dioxocyclohexane $(\mathbf{8 g})$, and 1,3-dioxocyclopentane $(\mathbf{8 h})$ were carried out under the conditions shown in Table 1 and were similarly treated to give 1[cyano(methoxycarbonyl)methylene]-1,2-dihydro-2-methyl-isoquinoline (9b), 1-[bis(cyano)methylene]-1,2-dihydro-2-methylisoquinoline (9c), 1[acetyl(methoxy-carbonyl)methylene]-1,2-dihydro-2-methylisoquinoline (9d), 1-[bis(acetyl)methylene]-1,2-dihydro-2-methylisoquinoline (9e), 1[benzoyl(ethoxycarbonyl)methylene]-1,2-dihydro-2-methylisoquinoline (9f), 1,2-dihydro-2-methyl-1-(2,6-dioxocyclohexylidene)isoquinoline $(\mathbf{9 g}), 1,2-$ dihydro-2-methyl-1-(2,5-dioxocyclopentylidene)isoquinoline (9h), 2-benzyl1,2-dihydro-1-[bis(methoxycarbonyl)methylene]isoquinoline (10a), 2-benzyl-1,2-dihydro-1-[cyano(methoxycarbonyl)methylene]isoquinoline (10b), 2-benzyl-1,2-dihydro-1-[bis(cyano)methylene] isoquinoline (10c), 1-[acetyl(methoxycarbonyl)methylene]-2-benzyl-1,2-dihydroisoquinoline (10d), and 1-[bis(acetyl)methylene]-2-benzyl-1,2-dihydroisoquinoline (10e). Yields are listed in Table 1.

9a: Yellow crystalline powder (acetone), mp $237-239^{\circ} \mathrm{C}$. IR $(\mathrm{KBr})$ $\mathrm{cm}^{-1}: 1720,1600 .{ }^{1} \mathrm{H}-\mathrm{NMR}\left(\mathrm{CDCl}_{3}\right) \delta: 3.62(6 \mathrm{H}, \mathrm{s}, \mathrm{OMe} \times 2), 4.22(3 \mathrm{H}, \mathrm{s}$, $\mathrm{NMe}), 7.66(1 \mathrm{H}, \mathrm{d}, J=7.0 \mathrm{~Hz}, \mathrm{H}-4), 7.72(1 \mathrm{H}, \mathrm{d}, J=2.0,6.0,8.0 \mathrm{~Hz}, \mathrm{H}-7)$, $7.84-7.87(2 \mathrm{H}, \mathrm{m}, \mathrm{H}-5,6), 7.90(1 \mathrm{H}, \mathrm{d}, J=7.0 \mathrm{~Hz}, \mathrm{H}-3), 8.50(1 \mathrm{H}, \mathrm{dd}$, $J=1.5,8.0 \mathrm{~Hz}, \mathrm{H}-8) .{ }^{13} \mathrm{C}-\mathrm{NMR}\left(\mathrm{CDCl}_{3}\right) \delta: 45.00,50.23(\mathrm{C} 2), 121.07$, $126.63,129.69,130.55,131.40,132.16,133.54,134.21,136.31,165.63$, 167.27 (C2). MS m/z: $273\left(\mathrm{M}^{+}\right)$. Anal. Calcd for $\mathrm{C}_{15} \mathrm{H}_{15} \mathrm{NO}_{4}$ : C, 65.92; H, 5.53 ; N, 5.13. Found: C, 65.56; H, 5.07; N, 5.60.

9b: Yellow needles (benzene), $\mathrm{mp} 194-196^{\circ} \mathrm{C}$. IR $(\mathrm{KBr}) \mathrm{cm}^{-1}: 2200$, 1660. ${ }^{1} \mathrm{H}-\mathrm{NMR}\left(\mathrm{CDCl}_{3}\right) \delta: 3.80(3 \mathrm{H}, \mathrm{s}, \mathrm{OMe}), 4.06(3 \mathrm{H}, \mathrm{s}, \mathrm{NMe}), 7.39(1 \mathrm{H}$, d, $J=7.1 \mathrm{~Hz}, \mathrm{H}-4), 7.65(1 \mathrm{H}, \mathrm{d}, J=7.1 \mathrm{~Hz}, \mathrm{H}-3), 7.70-7.86(3 \mathrm{H}, \mathrm{m}, \mathrm{H}-5,6$, 7), $8.93(1 \mathrm{H}, \mathrm{dd}, J=1.0,7.8 \mathrm{~Hz}, \mathrm{H}-8) .{ }^{13} \mathrm{C}-\mathrm{NMR}\left(\mathrm{CDCl}_{3}\right) \delta: 47.22,51.32$, $118.61,123.94,126.97,128.28,129.33,130.67,133.96,133.99,135.59$ (C2), 159.57, 167.77. MS m/z: $240\left(\mathrm{M}^{+}\right)$. Anal. Calcd for $\mathrm{C}_{14} \mathrm{H}_{12} \mathrm{~N}_{2} \mathrm{O}_{2}$ : C, 69.99; H, 5.03; N, 11.66. Found: C, 69.77; H, 4.94; N, 11.65.

9c: Yellow needles (acetone-chloroform), mp 226-227 ${ }^{\circ} \mathrm{C}$. IR ( $\left.\mathrm{KBr}\right)$ $\mathrm{cm}^{-1}: 2200,1630 .{ }^{1} \mathrm{H}-\mathrm{NMR}\left(\mathrm{CF}_{3} \mathrm{COOD}\right) \delta: 4.11(3 \mathrm{H}, \mathrm{s}, \mathrm{NMe}), 7.10(1 \mathrm{H}, \mathrm{d}$, $J=7.3 \mathrm{~Hz}, \mathrm{H}-4), 7.32(1 \mathrm{H}, \mathrm{d}, J=7.3 \mathrm{~Hz}, \mathrm{H}-3), 7.65-7.84(3 \mathrm{H}, \mathrm{m}, \mathrm{H}-5,6,7)$, $9.12(1 \mathrm{H}, \mathrm{dd}, J=1.0,8.1 \mathrm{~Hz}, \mathrm{H}-8) .{ }^{13} \mathrm{C}-\mathrm{NMR}\left(\mathrm{CDCl}_{3}\right) \delta: 47.51,116.00$, 120.04 (C2), 125.91, 127.16, 128.53, 129.09, 133.89 (C2), 134.80 (C2), 158.17. MS: $m / z: 207\left(\mathrm{M}^{+}\right)$. Anal. Calcd for $\mathrm{C}_{13} \mathrm{H}_{9} \mathrm{~N}_{3}: \mathrm{C}, 75.34 ; \mathrm{H}, 4.38 ; \mathrm{N}$, 20.28. Found: C, 75.28; H, 4.56; N, 20.31.

9d: Yellow crystalline powder (acetone), mp $224-225^{\circ} \mathrm{C}$. IR $(\mathrm{KBr})$ $\mathrm{cm}^{-1}: 1660,1650 .{ }^{1} \mathrm{H}-\mathrm{NMR}\left(\mathrm{CDCl}_{3}\right) \delta: 2.62(3 \mathrm{H}, \mathrm{s}, \mathrm{COMe}), 3.44(3 \mathrm{H}, \mathrm{s}$, $\mathrm{NMe}), 4.22(3 \mathrm{H}, \mathrm{s}, \mathrm{OMe}), 7.69-7.78(1 \mathrm{H}, \mathrm{br} \mathrm{m}, \mathrm{H}$-aromatic), $7.71(1 \mathrm{H}, \mathrm{d}$, $J=7.3 \mathrm{~Hz}, \mathrm{H}-4), 7.84-7.92(2 \mathrm{H}, \mathrm{m}, \mathrm{H}$-aromatic), $7.95(1 \mathrm{H}, \mathrm{d}, J=7.3 \mathrm{~Hz}, \mathrm{H}-$ 3), $8.45(1 \mathrm{H}, \mathrm{dd}, J=1.0,8.6 \mathrm{~Hz}, \mathrm{H}-8) .{ }^{13} \mathrm{C}-\mathrm{NMR}\left(\mathrm{CDCl}_{3}\right) \delta: 28.44,45.83$, $49.74,118.55,121.85,126.66,129.90,131.00,131.93,133.65,134.49$, 136.47, 167.00, 167.66, 190.04. MS m/z: $257\left(\mathrm{M}^{+}\right)$. Anal. Calcd for $\mathrm{C}_{15} \mathrm{H}_{15} \mathrm{NO}_{3}: \mathrm{C}, 70.02 ; \mathrm{H}, 5.88 ; \mathrm{N}, 5.44$. Found: $\mathrm{C}, 69.90 ; \mathrm{H}, 5.97 ; \mathrm{N}, 5.50$.

9e: Yellow crystalline powder (acetone), mp $210-211^{\circ} \mathrm{C}$. IR ( $\mathrm{KBr}$ ) $\mathrm{cm}^{-1}: 1670,1640 .{ }^{1} \mathrm{H}-\mathrm{NMR}\left(\mathrm{CF}_{3} \mathrm{COOD}\right) \delta: 1.95(6 \mathrm{H}, \mathrm{s}, \mathrm{COMe} \times 2), 4.54$ $(3 \mathrm{H}, \mathrm{s}, \mathrm{NMe}), 8.20(1 \mathrm{H}, \mathrm{dd}, J=7.6,7.6 \mathrm{~Hz}, \mathrm{H}-6$ or 7$), 8.34-8.39(3 \mathrm{H}, \mathrm{m}$, H-aromatic), $8.51(1 \mathrm{H}, \mathrm{d}, J=6.8 \mathrm{~Hz}, \mathrm{H}-3$ or 4$), 8.67(1 \mathrm{H}, \mathrm{d}, J=6.8 \mathrm{~Hz}, \mathrm{H}-3$ or 4$) .{ }^{13} \mathrm{C}-\mathrm{NMR}\left(\mathrm{CF}_{3} \mathrm{COOD}\right) \delta: 25.54(\mathrm{C} 2), 48.81,107.62,130.12,130.67$, 131.18, 132.31, 136.36, 139.08, 140.78, 141.72, 157.94, 196.13 (C2). MS $\mathrm{m} / \mathrm{z}: 241\left(\mathrm{M}^{+}\right)$. HR-MS $\mathrm{m} / \mathrm{z}$ : Calcd for $\mathrm{C}_{15} \mathrm{H}_{15} \mathrm{NO}_{2}, 241.1109$. Found: 241.1111.

9f: Yellow crystalline powder (benzene), mp $175-178^{\circ} \mathrm{C}$. IR $(\mathrm{KBr})$ $\mathrm{cm}^{-1}: 1640,1620 .{ }^{1} \mathrm{H}-\mathrm{NMR}\left(\mathrm{CDCl}_{3}\right) \delta: 0.85(3 \mathrm{H}, \mathrm{dd}, J=8.0,8.0 \mathrm{~Hz}, \mathrm{CMe})$, $3.38\left(2 \mathrm{H}\right.$, ddd, $\left.J=8.0,8.0,8.0 \mathrm{~Hz}, \mathrm{COOCH}_{2}\right), 4.16(3 \mathrm{H}, \mathrm{s}, \mathrm{NMe}), 7.16-$ $8.85(10 \mathrm{H}, \mathrm{m}, \mathrm{H}-3,4,5,6,7, \mathrm{Ph}), 8.32-8.60(1 \mathrm{H}, \mathrm{m}, \mathrm{H}-8) .{ }^{13} \mathrm{C}-\mathrm{NMR}$ $\left(\mathrm{CDCl}_{3}\right) \delta: 14.06,45.93,58.52,91.11,121.18,126.73,127.40$ (C2), 127.45 (C2), 128.54, 129.71, 130.81, 131.81, 133.79, 134.21, 136.34, 144.35, 166.04, 168.12, 188.91. MS m/z: : $333\left(\mathrm{M}^{+}\right)$. Anal. Calcd for $\mathrm{C}_{21} \mathrm{H}_{19} \mathrm{NO}_{3}: \mathrm{C}$, 75.65; H, 5.74; N, 4.20. Found: C, 75.61; H, 6.00; N, 4.17.

9g: Pale yellow crystalline powder (benzene), $\mathrm{mp} 230-233^{\circ} \mathrm{C}$. IR $(\mathrm{KBr})$ $\mathrm{cm}^{-1}: 1620,1600,820,750 .{ }^{1} \mathrm{H}-\mathrm{NMR}\left(\mathrm{CDCl}_{3}\right) \delta: 2.00-2.40\left(2 \mathrm{H}, \mathrm{m}, \mathrm{CH}_{2}\right)$, 
$2.66-2.79\left(4 \mathrm{H}, \mathrm{m}, \mathrm{COCH}_{2} \times 2\right), 4.23(3 \mathrm{H}, \mathrm{s}, \mathrm{NMe}), 7.73(1 \mathrm{H}, \mathrm{d}, J=7.0 \mathrm{~Hz}$, $\mathrm{H}-4), 7.72-8.00(3 \mathrm{H}, \mathrm{m}, \mathrm{H}-5,6,7), 7.96(1 \mathrm{H}, \mathrm{d}, J=7.0 \mathrm{~Hz}, \mathrm{H}-3), 8.43(1 \mathrm{H}$, br m, H-8) ${ }^{13} \mathrm{C}-\mathrm{NMR}\left(\mathrm{CDCl}_{3}\right) \delta: 21.52,37.13(\mathrm{C} 2), 46.09,105.84,122.62$, $126.76,129.89,130.15,132.35,133.97,134.95,136.47,165.31,192.17$ (C2). MS m/z: $253\left(\mathrm{M}^{+}\right)$. Anal. Calcd for $\mathrm{C}_{16} \mathrm{H}_{15} \mathrm{NO}_{2}: \mathrm{C}, 75.87 ; \mathrm{H}, 5.97 ; \mathrm{N}$, 5.53. Found: C, 75.44; H, 6.02; N, 5.44.

9h: Yellow needles (benzene), $\mathrm{mp} 291-293^{\circ} \mathrm{C}$. IR $(\mathrm{KBr}) \mathrm{cm}^{-1}: 1625$, $1600,810,755 .{ }^{1} \mathrm{H}-\mathrm{NMR}\left(\mathrm{CDCl}_{3}\right) \delta: 2.73\left(4 \mathrm{H}, \mathrm{s}, \mathrm{COCH}_{2} \times 2\right), 4.33(3 \mathrm{H}, \mathrm{s}$, $\mathrm{NMe}), 7.50-8.06(5 \mathrm{H}, \mathrm{m}, \mathrm{H}-3,4,5,6,7), 8.43(1 \mathrm{H}, \mathrm{m}, \mathrm{H}-8) .{ }^{13} \mathrm{C}-\mathrm{NMR}$ $\left(\mathrm{CDCl}_{3}\right) \delta: 34.48(\mathrm{C} 2), 46.83,106.45,121.69,126.68,128.46,129.82$, 132.66, 134.21, 135.02, 136.31, 159.89, $199.66(\mathrm{C} 2)$. MS $m / z: 239\left(\mathrm{M}^{+}\right)$. Anal. Calcd for $\mathrm{C}_{15} \mathrm{H}_{13} \mathrm{NO}_{2}:$ C, 75.30; H, 5.48; N, 5.87. Found: C, 75.03; H, 5.34; N, 5.87 .

10a: Red plates (acetone), $\mathrm{mp} 239^{\circ} \mathrm{C}$. IR $(\mathrm{KBr}) \mathrm{cm}^{-1}: 1701,1595,758$. ${ }^{1} \mathrm{H}-\mathrm{NMR}\left(\mathrm{CDCl}_{3}\right) \delta: 3.63(6 \mathrm{H}, \mathrm{s}, \mathrm{OMe} \times 2), 5.76\left(2 \mathrm{H}, \mathrm{s}, \mathrm{CH}_{2}\right), 7.25(2 \mathrm{H}, \mathrm{dd}$, $J=2.0,6.6 \mathrm{~Hz}, \mathrm{H}-\mathrm{Ph}), 7.32-7.39(3 \mathrm{H}, \mathrm{m}, \mathrm{H}-\mathrm{Ph}), 7.58(1 \mathrm{H}, \mathrm{d}, J=7.0 \mathrm{~Hz}$, $\mathrm{H}-4), 7.72-7.88(4 \mathrm{H}, \mathrm{m}, \mathrm{H}-3,5,6,7), 8.60(1 \mathrm{H}, \mathrm{d}, J=8.2 \mathrm{~Hz}, \mathrm{H}-8) .{ }^{13} \mathrm{C}-$ NMR $\left(\mathrm{CDCl}_{3}\right) \delta: 50.10(\mathrm{C} 2), 60.49,121.38,126.63(\mathrm{C} 2), 128.95,129.04$, 129.30 (C3), 129.81, 131.34, 132.26, 132.69, 134.54 (C2), 134.90, 136.33, 166.25, 167.24. MS m/z: $349\left(\mathrm{M}^{+}\right)$, 230. HR-MS $m / z$ : Calcd for $\mathrm{C}_{21} \mathrm{H}_{19} \mathrm{NO}_{4}$, 349.1314. Found: 349.1283 .

10b: Red needles (acetone), mp $91-93^{\circ} \mathrm{C}$. IR $(\mathrm{KBr}) \mathrm{cm}^{-1}: 2172,1713$, 1643, 756. ${ }^{1} \mathrm{H}-\mathrm{NMR}\left(\mathrm{CDCl}_{3}\right) \delta: 3.81(3 \mathrm{H}, \mathrm{s}, \mathrm{OMe}), 5.77\left(2 \mathrm{H}, \mathrm{s}, \mathrm{CH}_{2}\right)$, $7.17-7.24(2 \mathrm{H}, \mathrm{m}, \mathrm{H}-\mathrm{Ph}), 7.34-7.39(4 \mathrm{H}, \mathrm{m}, \mathrm{H}-4, \mathrm{Ph}), 7.50(1 \mathrm{H}, \mathrm{d}$, $J=7.2 \mathrm{~Hz}, \mathrm{H}-3), 7.70-7.87(3 \mathrm{H}, \mathrm{m}, \mathrm{H}-5,6,7), 9.09(1 \mathrm{H}, \mathrm{d}, J=8.9 \mathrm{~Hz}, \mathrm{H}-8)$. ${ }^{13} \mathrm{C}-\mathrm{NMR}\left(\mathrm{CDCl}_{3}\right) \delta: 51.29,61.92,118.47,124.12,127.02(\mathrm{C} 2), 128.28$, 128.50, 129.11 (C2), 129.42 (C2), 130.66, 132.56, 134.16, 135.08, 135.31 (C2), 139.87, 168.09. MS m/z: : $316\left(\mathrm{M}^{+}\right), 285,257,91$. HR-MS $m / z$ : Calcd for $\mathrm{C}_{20} \mathrm{H}_{16} \mathrm{~N}_{2} 0_{2}, 316.1212$. Found: 316.1248 .

10c: Yellow needles (acetone), mp $215-216^{\circ} \mathrm{C}$. IR $(\mathrm{KBr}) \mathrm{cm}^{-1}: 2191$, 2162, 1629, 732. ${ }^{1} \mathrm{H}-\mathrm{NMR}\left(\mathrm{CDCl}_{3}\right) \delta: 5.62\left(2 \mathrm{H}, \mathrm{s}, \mathrm{CH}_{2}\right), 7.00(1 \mathrm{H}, \mathrm{d}$, $J=7.0 \mathrm{~Hz}, \mathrm{H}-4), 7,18-7.26$ (3H, m, H-3, Ph), 7.38-7.44 (3H, m, H-Ph), $7.65-7.72(2 \mathrm{H}, \mathrm{m}, \mathrm{H}-5,6), 7.80(1 \mathrm{H}, \mathrm{dd}, J=1.0,7.6 \mathrm{~Hz}, \mathrm{H}-7), 9.13(1 \mathrm{H}, \mathrm{d}$, $J=8.6 \mathrm{~Hz}, \mathrm{H}-8) .{ }^{13} \mathrm{C}-\mathrm{NMR}\left(\mathrm{CDCl}_{3}\right) \delta: 61.42,115.60,119.98,126.15$, $127.13,128.36,128.53,128.97,129.11,129.42,132.83$ (C5), 133.89, 134.00, 134.63, 158.54. MS: $m / z: 283\left(\mathrm{M}^{+}\right)$. Anal. Calcd for C, 80.54; N, 14.83; H, 4.63. Found: C, 80.61; N, 14.75; H, 4.91 .

10d: Yellow plates (acetone), $\mathrm{mp} 250^{\circ} \mathrm{C}$. IR $(\mathrm{KBr}) \mathrm{cm}^{-1}: 1733,1716$, 1655, 772. ${ }^{1} \mathrm{H}-\mathrm{NMR}\left(\mathrm{CDCl}_{3}\right) \delta: 2.65(3 \mathrm{H}, \mathrm{s}, \mathrm{COMe}), 3.43(3 \mathrm{H}, \mathrm{s}, \mathrm{OMe})$ $5.55(1 \mathrm{H}, \mathrm{d}, J=15.0 \mathrm{~Hz}, \mathrm{CH}-\mathrm{Ph}), 5.94(1 \mathrm{H}, \mathrm{d}, J=15.0 \mathrm{~Hz}, \mathrm{CH}-\mathrm{Ph}), 7.25$ $(5 \mathrm{H}, \mathrm{s}, \mathrm{H}-\mathrm{Ph}), 7.66(1 \mathrm{H}, \mathrm{d}, J=7.1 \mathrm{~Hz}, \mathrm{H}-4), 7.72-7.92(4 \mathrm{H}, \mathrm{m}, \mathrm{H}-3,5,6$, 7), $8.53(1 \mathrm{H}, \mathrm{d}, J=8.4 \mathrm{~Hz}, \mathrm{H}-8) .{ }^{13} \mathrm{C}-\mathrm{NMR}\left(\mathrm{CDCl}_{3}\right) \delta: 28.37,49.65,60.52$, $122.06,126.72,128.92(\mathrm{C} 2), 129.11(\mathrm{C} 2), 129.31,130.02,130.84(\mathrm{C} 2)$, 132.42, 134.02 (C2), 130.84, 136.56 (C2), 167.60, 190.13. MS m/z: 333 $\left(\mathrm{M}^{+}\right)$, 274, 260, 91. HR-MS $m / z$ : Calcd for $\mathrm{C}_{21} \mathrm{H}_{19} \mathrm{NO}_{3}, 333.1365$. Found: 333.1378 .

10e: Yellow plates (acetone), $\mathrm{mp} 190^{\circ} \mathrm{C} . \mathrm{IR}\left(\mathrm{CHCl}_{3}\right) \mathrm{cm}^{-1}: 1735,1718$, 1699, 1629, 772, 686. ${ }^{1} \mathrm{H}-\mathrm{NMR}\left(\mathrm{CDCl}_{3}\right) \delta: 2.17(6 \mathrm{H}, \mathrm{s}, \mathrm{COMe} \times 2), 5.74$ $\left(2 \mathrm{H}, \mathrm{s}, \mathrm{CH}_{2}\right), 7.30-7.35(2 \mathrm{H}, \mathrm{m}, \mathrm{H}-\mathrm{Ph}), 7.36-7.41(3 \mathrm{H}, \mathrm{m}, \mathrm{H}-\mathrm{Ph}), 7.71-$ $7.82(2 \mathrm{H}, \mathrm{m}, \mathrm{H}$-aromatic), $7.86-7.94(3 \mathrm{H}, \mathrm{m}, \mathrm{H}$-aromatic $), 8.57(1 \mathrm{H}, \mathrm{d}$, $J=8.6 \mathrm{~Hz}, \mathrm{H}-8) .{ }^{13} \mathrm{C}-\mathrm{NMR}\left(\mathrm{CDCl}_{3}\right) \delta: 29.65,30.88,60.42,122.27,126.84$, 129.16 (C2), 129.43 (C3), 129.46, 132.28 (C2), 132.47, 134.17, 135.20, 136.70 (C2), 169.98, 196.22 ( C2). MS $m / z: 317\left(\mathrm{M}^{+}\right), 274,260,232$. HRMS $m / z$ : Calcd for $\mathrm{C}_{21} \mathrm{H}_{19} \mathrm{NO}_{2}, 317.1416$. Found: 317.1387 .

General Procedure for Reaction of 10a,d,e with Acetic Anhydride A mixture of 10a $(0.349 \mathrm{~g}, 1.0 \mathrm{mmol})$ and acetic anhydride $(4 \mathrm{ml})$ was refluxed for $4 \mathrm{~h}$ and concentrated in vavuo. The residue was dissolved in water $(5 \mathrm{ml})$, and the resulting solution was extracted with $\mathrm{CHCl}_{3}$. The organic layer was dried over $\mathrm{MgSO}_{4}$, and concentrated in vacuo. The residue was purified using column chromatography on aluminum oxide $\left(\mathrm{CHCl}_{3}\right.$-acetone, $2: 1)$ to give 2-acetoxy-1-methoxycarbonyl-3-phenylpyrrolo[2-1-a]isoquinoline $[11 \mathbf{a}(0.357 \mathrm{~g}, 99 \%)]$. Reactions of 10d, e with acetic anhydride were similarly performed to give 2-acetoxy-1-acetyl-3-phenylpyrrolo[2-1-a]isoquinoline (11b), and 1-acetyl-2-methyl-3-phenylpyrrolo[2-1-a] isoquinoline (11c). Yields are listed in Chart 3.

11a: Yellow needles $\left(\mathrm{Et}_{2} \mathrm{O}\right), \mathrm{mp} 165-166^{\circ} \mathrm{C}$. IR $\left(\mathrm{CHCl}_{3}\right) \mathrm{cm}^{-1}: 1764$, 1702, 1606. ${ }^{1} \mathrm{H}-\mathrm{NMR}\left(\mathrm{CDCl}_{3}\right) \delta: 2.25(3 \mathrm{H}, \mathrm{s}, \mathrm{COMe}), 3.95(3 \mathrm{H}, \mathrm{s}$, COOMe), $6.93(1 \mathrm{H}, \mathrm{d}, J=7.4 \mathrm{~Hz}, \mathrm{H}-6), 7.45-7.60(8 \mathrm{H}, \mathrm{m}, \mathrm{H}-7,8,9, \mathrm{Ph})$, $7.83(1 \mathrm{H}, \mathrm{d}, J=7.4 \mathrm{~Hz}, \mathrm{H}-5), 9.40(1 \mathrm{H}, \mathrm{d}, J=8.0 \mathrm{~Hz}, \mathrm{H}-10) .{ }^{13} \mathrm{C}-\mathrm{NMR}$ $\left(\mathrm{CDCl}_{3}\right) \delta: 20.64,51.60,101.45,113.71,119.18,121.32,125.40,126.63$, $126.92,127.62,127.65,127.81,127.84,128.83,129.03,129.15,129.23$, 129.81, 130.08, 137.31, 164.77, 170.01. MS: $\mathrm{m} / \mathrm{z}: 359\left(\mathrm{M}^{+}\right), 317,285,228$. HR-MS $m / z$ : Calcd for $\mathrm{C}_{22} \mathrm{H}_{17} \mathrm{NO}_{4}, 359.1158$. Found: 359.1187 .

11b: Yellow plates (acetone), mp $145-146^{\circ} \mathrm{C}$. IR $\left(\mathrm{CHCl}_{3}\right) \mathrm{cm}^{-1}: 1764$, $1718,1700,1605 .{ }^{1} \mathrm{H}-\mathrm{NMR}\left(\mathrm{CDCl}_{3}\right) \delta: 2.22(3 \mathrm{H}, \mathrm{s}, \mathrm{COMe}), 2.63(3 \mathrm{H}, \mathrm{s}$, OCOMe), $6.95(1 \mathrm{H}, \mathrm{dd}, J=0.7,7.3 \mathrm{~Hz}, \mathrm{H}-6), 7.46-7.63(8 \mathrm{H}, \mathrm{m}, \mathrm{H}-7,8,9$, $\mathrm{Ph}), 7.80(1 \mathrm{H}, \mathrm{d}, J=7.3 \mathrm{~Hz}, \mathrm{H}-5), 9.10(1 \mathrm{H}, \mathrm{ddd}, J=0.7,1.4,6.4 \mathrm{~Hz}, \mathrm{H}-10)$. ${ }^{13} \mathrm{C}-\mathrm{NMR}\left(\mathrm{CDCl}_{3}\right) \delta: 20.82,31.10,111.82,114.10,119.05,121.16,125.44$, $126.18,126.98,127.59,127.88,127.91,128.68,128.94,129.11,129.23$ (C2), 130.03 (C2), 136.88, 169.43, 194.95. MS: $m / z: 343\left(\mathrm{M}^{+}\right), 301,286$. HR-MS m/z: Calcd for $\mathrm{C}_{22} \mathrm{H}_{17} \mathrm{NO}_{4}, 359.1158$. Found: 359.1187 .

11c: Colorless oil. IR $\left(\mathrm{CHCl}_{3}\right) \mathrm{cm}^{-1}: 1718,1655,1604 .{ }^{1} \mathrm{H}-\mathrm{NMR}\left(\mathrm{CDCl}_{3}\right)$ $\delta: 2.33(3 \mathrm{H}, \mathrm{s}, \mathrm{COMe}), 2.71(3 \mathrm{H}, \mathrm{s}, \mathrm{Me}), 6.78(1 \mathrm{H}, \mathrm{dd}, J=0.8,7.3 \mathrm{~Hz}, \mathrm{H}-6)$, $7.39-7.56(8 \mathrm{H}, \mathrm{m}, \mathrm{H}-7,8,9, \mathrm{Ph}), 7.68(1 \mathrm{H}, \mathrm{d}, J=7.3 \mathrm{~Hz}, \mathrm{H}-5), 8.52(1 \mathrm{H}$, dd, $J=0.8,8.3 \mathrm{~Hz}, \mathrm{H}-10) .{ }^{13} \mathrm{C}-\mathrm{NMR}\left(\mathrm{CDCl}_{3}\right) \delta: 12.11,32.39,112.42$, $119.74,120.89,121.77,125.04,125.26,126.21,126.90,126.93,127.35$, $128.41,128.69,128.85,129.04$ (C2), 130.53, 130.94 (C2), 200.30. MS: $m / z$ : $299\left(\mathrm{M}^{+}\right)$, 284. HR-MS $\mathrm{m} / \mathrm{z}$ : Calcd for $\mathrm{C}_{21} \mathrm{H}_{17} \mathrm{NO}, 299.1310$. Found: 299.1311 .

\section{References}

1) Tomisawa H., Tanbara T., Kato H., Hongo H., Fujita R., Heterocycles, 15, 277-280 (1981).

2) Iwao M., Kuraishi T., Bull. Chem. Soc. Jpn., 53, 297-298 (1980); Review of synthesis of pyrrolo[2,1-a]isoquinolines: Uchida T., Matsumoto K., Synthesis, 1976, 209-236.

3) Ishibashi F., Miyazaki Y., Iwao M., Tetrahedron, 53, 5951-5962 (1997); Lindquist N., Fenical W., Van Duyne G. D., Clardy J., J. Org. Chem., 53, 4570 - 4574 (1988); Carroll A. R., Bowden B. F., Coll J. C., Aust. J. Chem., 46, 489-501 (1993).

4) Boekelheide V., Godfrey J. C., J. Am. Chem. Soc., 75, 3679-3685 (1953).

5) Huisgen R., Grashey R., Steingruber E., Tetrahedron Lett., 1963, 1441-1445; Kobayashi Y., Kumadaki I., Sekine Y., Kutsuma T., Chem. Pharm. Bull., 21, 1118-1123 (1973); Basketter N. S., Plunkett A. O., J. Chem. Soc., Chem. Comm., 1973, 188-189.

6) Krohnke F., Zecher W., Chem. Ber., 95, 1128-1137 (1962).

7) Tomisawa H., Wang C. H., Chem. Pharm. Bull., 21, 2607-2612 (1973).

8) Tomisawa H., Saito K., Hongo H., Fujita R., Chem. Pharm. Bull., 18, 937-940 (1970); Tomisawa H., Fujita R., ibid., 21, 2585-2589 (1973); Tomisawa H., Fujita R., Hongo H., Kato H., ibid., 22, 20912096 (1974).

9) Barlin G. B., Benbow J. A., J. Chem. Soc. Perkin Trans. II., 1975, $298-302$. 